\title{
Characterization of Tribofilm from Sulfurized Mohwa Oil
}

\author{
Arun Kumar Singh \\ Chemical Science Division, Indian Institute of Petroleum (CSIR), Dehradun 248005, India \\ Correspondence should be addressed to Arun Kumar Singh, arun@iip.res.in
}

Received 11 July 2011; Accepted 14 August 2011

Academic Editors: M. Ahmadian and C. J. Ho

Copyright (@ 2011 Arun Kumar Singh. This is an open access article distributed under the Creative Commons Attribution License, which permits unrestricted use, distribution, and reproduction in any medium, provided the original work is properly cited.

\begin{abstract}
The reaction products of sulfurized Mohwa oil with iron powder in hydrocarbon medium at $150^{\circ} \mathrm{C}$ for $8 \mathrm{~h}$ were studied to investigate the type of lubricant films formed during their application as antiwear and extreme pressure additives. The main reaction product was isolated on the basis of its solubility in mixed solvent. Surface characterization was carried out using ultraviolet-visible spectroscopy (UV), fourier transform infrared spectroscopy (FTIR), nuclear magnetic resonance (NMR), X-ray diffraction spectroscopy (XRD), scanning electron microscope (SEM), and high frequency reciprocal rig (HFRR). An examination of their elemental analysis and instrumental analysis data reveals that there is reduction in the length of the alkyl chains and carbonyl ester groups with formation of inorganic iron sulfides. Polymerized product with a number of ketonic and aldehydic groups containing iron and sulfur in the polymeric films in the form of unsaturated cyclic rings was also formed. The films are organo-inorganic in nature, unlike the purely inorganic iron sulfide type. The load-carrying characteristic of this product is strongly influenced by the type of the film formed on the iron surfaces.
\end{abstract}

\section{Introduction}

The development of coating and lubricating materials for the surface modification, functionalization of metallic surfaces has become one of the major areas of research. Since the functionalization of iron ( $\mathrm{Fe}$ ) surface also depends upon the reactivity of the surface and strength of organic species. In addition, the surface functionalization is also the function of thermal stress, mechanical stress, catalytic activity, and the emissions of low exoelectrons. Functionalization of iron $(\mathrm{Fe})$ surface in the chemical environment of oxygen, chlorine, nitrogen, aluminum, boron, nickel, and carbon has been studied earlier also. However, study of the sulfur, phosphorus, and many other chemical species has been scanty in literature. The application range of organic sulfides is spread throughout the chemical, pharmaceutical, medicinal, fertilizers, electrical, electronic, biological, and biochemical sectors of scientific and technological appliances. These are directly or indirectly involved in most of the industrial, research, and domestic application.

In the present paper, it is aimed to conduct the model study for synthesis of organic sulfides and the surface functionalization of metallic iron $(\mathrm{Fe})$ particles in the chemical environment of organic sulfides for their industrial application in lubricating fluid industry/metal working fluid industry, coating industry, paint industry, and the industry for the automotive and industrial lubricating fluids and its formulations protocol. The concept of functionalities can also be applicable to different categories of research and analytical studies such as process optimization, material conservation, energy utilization, environmental pollution, natural diseases, metrological devices, medical appliances, surface/interfacial reaction, and petroleum exploration.

However, the concept of layer development and their microanalysis appeared more explicitly in the late eighties. Kajdas [1] reported the formation of organometallic compounds by the reaction of organic compounds onto the metallic surfaces and the subsequent reaction products as an effect of destruction in tribochemical environment follows as shown below:

Organic compounds

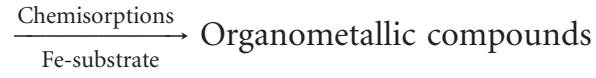


$\stackrel{\text { Chemical reaction }}{\longrightarrow}$ Complex compounds

Destruction high temperature Organic reactions

Exo electrons + Mechanical stress + Catalytic activity + Thermal impacts $\rightarrow$ Surface reactivity.

Under the conditions of heavy loads and high sliding speed the extreme pressure (EP) zone formed at metal-tometal junction. The mechanism of lubrication under the EP conditions by sulfurized additives is said to depend on the formation of a film of iron sulfide due to controlled chemical reactions of these additives with the rubbing surfaces. This reduces the wear and damage to rubbing surfaces [2-5]. Many studies have been reported on the chemical nature, elemental compositions, and kinetics of the EP films formed by the sulfur-bearing additives [6-10].

According to Allum and Ford [11] and Forbes [12], the load-carrying capacity provided by the lubricant film formed by the sulfurized additives depends upon the ease of cleavage of C-S bonds in the molecule, in the formation of inorganic iron sulfide on the rubbing surface. The performance of different sulfides depends upon the functional groups attached to the sulfide linkage. The diallyl and dibenzyl disulfides are the most effective EP additives because of the ease of formation of inorganic iron sulfide on account of the stability of the allyl and benzyl radicals $[11,12]$.

It was pointed out that the formation of organicinorganic films is chiefly responsible for providing the EP characteristics [13]. Several sulfides having sulfur in the allylic position show wide range of load carrying capacities and the EP film formed is distinctly organo-inorganic in nature. The inorganic iron sulfide layer does not seem to be the only part contributing to load carrying properties. This has leaded us to undertake studies on the reaction products of iron with sulfurized EP additives to investigate the chemical nature of the film formed. In this paper we describe studies relating to the various film-like reaction products of sulfurized Mohwa oil with iron powder conditions close to those prevailing during EP lubrication.

The surface films formation is important to protect the surfaces. There are three ways of film formation: physical adsorption, chemisorptions, and chemical reaction. The surface behavior of films is due to energy binding of the film molecules to the surface. Physical adsorption involves intermolecular forces involved in condensation of vapors to liquids. Liquid molecules attached to the surfaces of the solids, and this provides a modest protection. Physical adsorption is usually rapid, reversible, and nonspecific. There is no electron transfer in this process. An example is of physical adsorption of sulfide on iron (Figure 1). Because of the weak bonding energies involved, physically adsorbed species are usually not very effective. Chemically adsorbed films are generally produced by adding oil and additive. These additives generally contain sulfur molecules, which exhibit great affinity for metals at their active ends. The usual configuration of these polar molecules resembles that of a carpet pile with the molecules standing perpendicular to the surface. Such form metal salt/sulfide. The sulfide film is dense because of

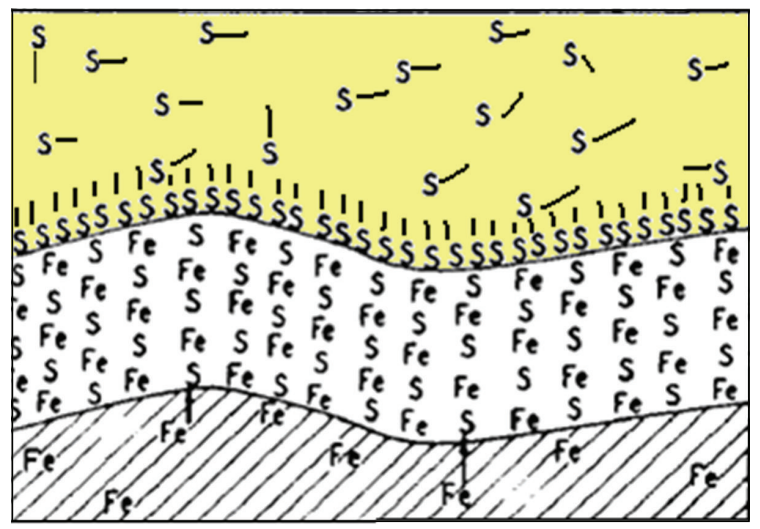

Figure 1: Adsorption of hexadecanol and sulfide.

the preferred orientation of the molecules. For example, on a steel surface, sulfide will form a monomolecular layer of iron sulfide. The effectiveness of these layers is limited by the melting point of the sulfide. Films formed by chemical reaction provide the greatest film strength and are used in the most severe operating conditions. It has already been noted that films formed by physical and chemical adsorption cease to be effective above certain transition temperatures, but high-melting-point sulfide survive at high temperatures. The formation of a chemical reaction film is specific, may be rapid or slow (depending on temperature, reactivity, and other conditions), and is irreversible [14]. Example of a reacted film of iron sulfide on an iron surface is shown in Figure 1.

The chemical analysis of the absorbed sulfide film indicates the purity of sulfides film around $98 \%$ with $2 \%$ of unreacted hydrocarbon, which is not detectable in the extraction stage of analysis. But on final analysis by sulfated ash, it was revealed that it is not possible to remove the entire unreacted hydrocarbon from sulfides. However, the correction for purity was made during the calculation and conclusion. Absence of inorganic content and water was an advantage. For characterization of the absorbed sulfide films the results of instrumental analysis, UV, XRD, SEM, IR and NMR, are important.

\section{Experimental}

2.1. Material. Electrolyte grade iron (Fe) particles (100-200 mish size), carrier fluid (light and medium paraffin's liquid). acetone, toluene, xylene, elemental (crystalline) sulfur powder $(99.9 \%)$, metal (alkali) sulfides $\left(\mathrm{M}_{2} \mathrm{~S}\right)$, heating system/oil bath arrangement., soxhlet apparatus arrangement, separating funnels, vacuum pump for filtration, vacuum oven (reduced pressure), evaporating arrangement, reflux arrangement.

\subsection{Methods}

2.2.1. Sulfurized Mohwa Oil. Crude Mohwa oil [15] is purified by dissolving in paraffinic solvent, cooling, and filtering. 


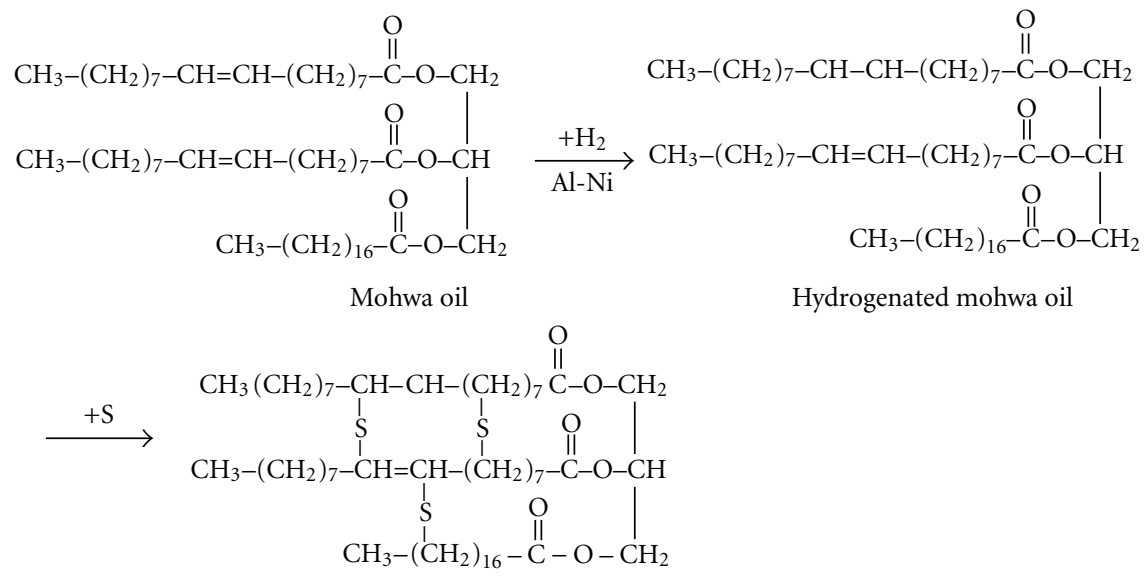

Sulfurized mohwa oil

SCHEME 1

The purified Mohwa oil is partially hydrogenated in the presence of solid catalyst (Raney nickel) and then sulfurized at elevated temperature. The product is purified by solvent treatment followed by copper treatment. In the process of synthesis 900 parts Mohwa oil and 10 parts sulfur were taken, and this mixture was stirred at $120^{\circ} \mathrm{C}$ for 3 hours after some time diluted with alcohol and filter it. The sulfurized Mohwa oil (SMO) was obtained. The following reaction and takes place in this process (Scheme 1).

2.2.2. Film Formation. The materials used involve Sulfurized Mohwa Oil (SMO), chloroform $\left(\mathrm{CHCl}_{3}\right)$, dimethylformamide $\left(\mathrm{C}_{3} \mathrm{H}_{7} \mathrm{ON}\right)$, xylene $\left(\mathrm{C}_{9} \mathrm{H}_{12}\right)$, n-heptane $\left(\mathrm{C}_{7} \mathrm{H}_{16}\right), 2 \mathrm{~N}$ hydrochloric acid $(\mathrm{HCl})$, electrolyte grade iron $(\mathrm{Fe})$ particles (100-200 mesh size powder) SD fine chemicals 38597, K-50 (Figure 2), and light paraffin liquid (sulfur-free). The slurry of $\mathrm{SMO}(5 \mathrm{wt} \%)$ and electrolyte grade iron (Fe) particle $(7 \mathrm{wt} \%)$ in light paraffin liquid was refluxed in a flask continuously for eighteen to twenty hours at temperature $150 \pm$ $5^{\circ} \mathrm{C}$. The reaction product was filtered and washed with petroleum solvent (bp $60^{\circ}-80^{\circ} \mathrm{C}$ ) until the trace of paraffin liquid was removed. The crude film was smoothly dried at reduced pressure and temperature to make it free from washing solvent. Further, it was treated with $2 \mathrm{~N}$-hydrochloric acid at the temperature slightly greater than room temperature and film was extracted by mix solvent [16]. The film specimen, namely, chemisorbed reaction (composite) film fraction, was subjected for analytical study like elemental composition, chemical composition, probable empirical formula as shown in Table 1 using FTIR, UV, XRD, NMR spectroscopic techniques and SEM microscopic technique for the identification of chemical moieties and functional groups.

\section{Analysis}

3.1. Elemental Analysis. The analysis and characterization of organic sulfides and chemisorbed reaction film (CRF). The organic sulfides are mainly characterized by elemental analysis. Elemental Analysis of organic sulfides takes place by

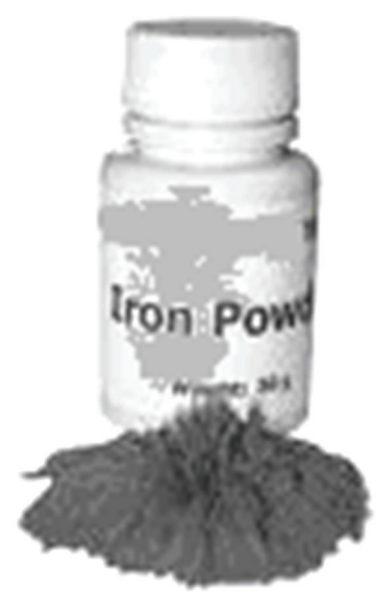

FIGURE 2: Electrolyte grade iron particles for reaction process.

Perkins Elmer Equipment (2400 series II CHNSO analyzer). This analysis describes the percentage, molecular weight, and Empirical formula of the organic compounds mainly organic sulfides. The percentages of carbon, hydrogen, sulfur, phosphorous oxygen, nitrogen, and iron are given in Table 1. The performances of SMO particularly copper strip corrosion, sulfur content, lubricity on high frequency reciprocal rig (HFRR) were measured and reported in Table 2.

3.2. Instrumental Analysis. In the present work, XRD spectrum of absorbed sulfide film was analyzed on Brucker Germany Powder XRD-D8 Advance. The XRD spectra as recorded are reproduced in Figure 3. Deferent types of peaks are obtained by XRD analysis of SMO. Peaks No1, 2 Theta 44.850 FWHM0.647 $d$-Value 2.0192 Intensity 60I/I 100 .

UV spectrum of absorbed sulfide film was analyzed on Lambda ULTRA-VIOLET VIS. N.K. SPECTROPHOTOMETER. The recorded UV spectrum is reproduced in Figure 4. As per analysis, monoaromatic was $38.7 \%$, diaromatic was 
TABLE 1: Elemental analysis, molecular weight, and empirical formula of SMO and SMO Film.

\begin{tabular}{lccccccc}
\hline & $\mathrm{C}$ & $\mathrm{H}$ & $\mathrm{S}$ & $\mathrm{O}$ & $\mathrm{Fe}$ & Molecular weight & Empirical formula \\
\hline SMO & 68.21 & 10.95 & 6.74 & 10.11 & - & 950 & $\mathrm{C}_{54} \mathrm{H}_{104} \mathrm{O}_{6} \mathrm{~S}_{2}$ \\
SMO Film & 58.12 & 9.22 & 5.74 & 8.6 & 18.32 & 1115 & $\mathrm{C}_{55} \mathrm{H}_{102.8} \mathrm{O}_{5.99} \mathrm{~S}_{2} \mathrm{Fe}_{3.64}$ \\
\hline
\end{tabular}

TABle 2: Product properties of sulfurized Mohwa oil.

\begin{tabular}{lc}
\hline Characteristics & Value \\
\hline Copper corrosion Test at $120^{\circ} \mathrm{C}$ for 3 hours & 1 a to $1 \mathrm{~b}$ \\
Sulfur, wt $\%$ & $12-13$ \\
Wear scar diameter on HFRR (in mm) & 0.408 \\
Friction coefficient on HFRR & 0.009 to 0.018 \\
Film's contact potential on HFRR (in mv) & 0.4 to 0.8 \\
Weld load $(\mathrm{Kg})$ & 455 \\
\hline
\end{tabular}

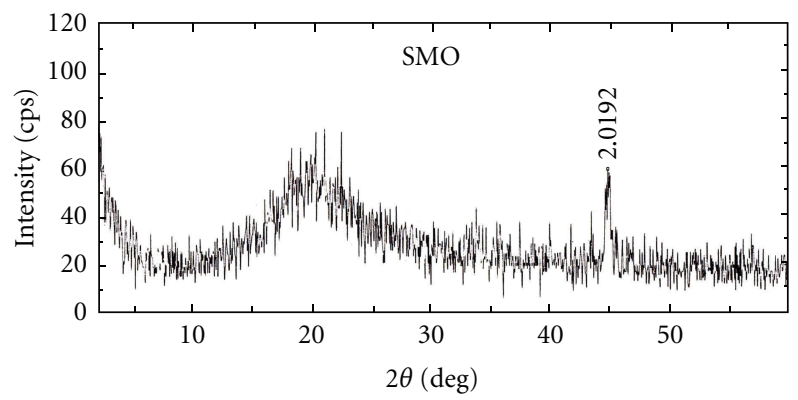

Figure 3: XRD Graph of SMO.

$8.2 \%$, polyaromatic was $16.5 \%$ of total aromatics, and total aromatic was $63.4 \%$ of reaction product.

FT-IR spectrum of absorbed sulfide film was run as either thin film between $\mathrm{KBr}$ plates or as $\mathrm{KBr}$ pellets from $4000 \mathrm{~cm}^{-1}$ to $400 \mathrm{~cm}^{-1}$ on Perkin Elmer 1760X FT-IR spectrometer. The recorded IR data are given in Figure 5: $3899 \mathrm{~cm}^{-1}$ to $3344 \mathrm{~cm}^{-1}[v(\mathrm{OH})], 2348$ to $2117 \mathrm{~cm}^{-1}\left[v(\mathrm{C}=\mathrm{O}), v_{\mathrm{as}}(\mathrm{C}=\mathrm{S})\right.$ aliphatic], $1742 \mathrm{~cm}^{-1}$ [ $v(\mathrm{C}=\mathrm{O}),(\mathrm{C}=\mathrm{S})$ quinoid], $1649 \mathrm{~cm}^{-1}$ Quniod, $1540-1514 \mathrm{~cm}^{-1} \quad v(\mathrm{C}=\mathrm{C}), \quad 1453 \mathrm{~cm}^{-1} \quad v(-\mathrm{OH})$, $1370-1170 \mathrm{~cm}^{-1} v(\mathrm{C}=\mathrm{O}), 1010-1020 \mathrm{~cm}^{-1} v(\mathrm{SO})$.

The ${ }^{1} \mathrm{H}$ NMR spectrum of sulfide was recorded on Bruker DRX 300 Spectrometer in $\mathrm{CdCl}_{3}$ solvent with TMS as internal standard. Signal positions ( $\delta$ values) were recorded along with frequencies and intensities of the signals. The spectrum is given in Figure 6. Carbon type to which the protons are attached and $\delta$ value in ppm $0.016,0.06,0.1,0.15,0.53,0.89$, 1 [Methyl $\mathrm{CH}_{3}$ ] $, 1.14,1.31,1.61,1.75,1.98$ ppm [Methylene $\mathrm{CH}_{2}$ ], 2.19, 2.29, $2.87 \mathrm{ppm}$ [Methylene S-CH $\mathrm{CH}_{2}$, $3.97 \mathrm{ppm}$ [Methylene Ac- $\mathrm{CH}_{2}$ ], 2.34-2.54 ppm [Methine $\mathrm{CH}$ ], $2.07 \mathrm{ppm}$ [Unreacted HC]. Carbon type to which the 13C are attached and $\delta$ value in ppm 13C-28.50, 28.66, 28.81, 28.97 ppm [Methyl $\mathrm{CH}_{3}$ ], 29.12, 29.27, 29.43 ppm [Hetero bond], $100.0 \mathrm{ppm}$ [aromatic bond], $205.29 \mathrm{ppm}$ [aromatic $\mathrm{C}-\mathrm{O}$ bond]. The 13C NMR spectrum is given in Figure 7.

SEM micrograph of absorbed sulfide film was run on Quanta 200F-FE-SEM-EDS-EESD system, FEI The Netherlands. The recorded SEM microanalyses are given in Figures 8 and 9. The SEM micrograph as recorded is reproduced in Figures 10 and 11. Energy value of SEM microanalysis at different places Approx. Energy eV $[\mathrm{KeV}=1000 \times \mathrm{eV}]$ at place 2.01-300, 500, 800, 2200, and at place 2.03-300, 500, 800, 2200,6500 .

\section{Result and Discussion}

It is not easy to predict the exact nature of SMO chemical reactions taking place between sulfide and iron. However, attempt has been made to increase the knowledge regarding reactions and extracted sulfide film, which was formed on iron surface. It is evident that in primary reactions, ionization occurs by capture of exoelectrons to form thiolate-type ions. Thermal decomposition of SMO produces R-S or R$\mathrm{S}_{2}$ radicals. Catalytic oxidation of hydrocarbon solvent gives aldehyde, ketones, acids, unsaturated hydrocarbons, polyketones, hydroxy ketones, carbanions, and conjugated ketonic and enolic species. In secondary reactions it occurs at the iron surface to form mercaptides. Other reactions are decomposition of radicals into further radicals, disproportionation of radicals to products and oxidative attack of thio radicals [R$\mathrm{S}$ ] on unsaturated hydrocarbons, ketones, aldehydes, acids, hydroxy ketones, polyketones, and so forth. Attack of reactive sulfur species on hydrocarbon solvent and other derivatives to produce olefinic derivatives. Cyclization to heterocyclic derivatives. Formation of iron sulfides by decomposition of mercaptides. Direct reaction with reactive sulfur and hydrogen abstraction from hydrocarbon solvent to form thiols [17].

Tertiary reactions occur at the iron surface to produce highly absorbed film-like organic species by interaction of ions, radicals, and other derivatives produced by primary and secondary reactions. More iron sulfide is formed by decomposition of mercaptides and reaction with sulfur-containing secondary reaction products. Several $\pi$-metal-type complexes and chelated iron derivatives of quinones and highly oxidized hydrocarbon solvent are formed.

The UV spectrum of SMO has lot of bending in curve. The important changes are at wavelength $(\lambda) 198,212$, and $220 \mathrm{~nm}$ and total calculated cyclic compounds are $63.4 \%$ out of which majority (38.7\%) portion is of monocyclic. SMO is an olefin compound and aromaticity in its molecule is not present. It can be inferred from UV analysis that SMO was decomposed significantly by thermochemical reaction on iron and a significant portion was cyclized and then adsorbed on iron surface.

Under chemical environment, sulfides reacted to iron surface with the help of exo-electron and catalytic effect of metal. The films formed may be indicator to the reactions taking place at the metal surface. An XRD spectrum of films generated from SMO is plotted in Figure 3. It can be seen 


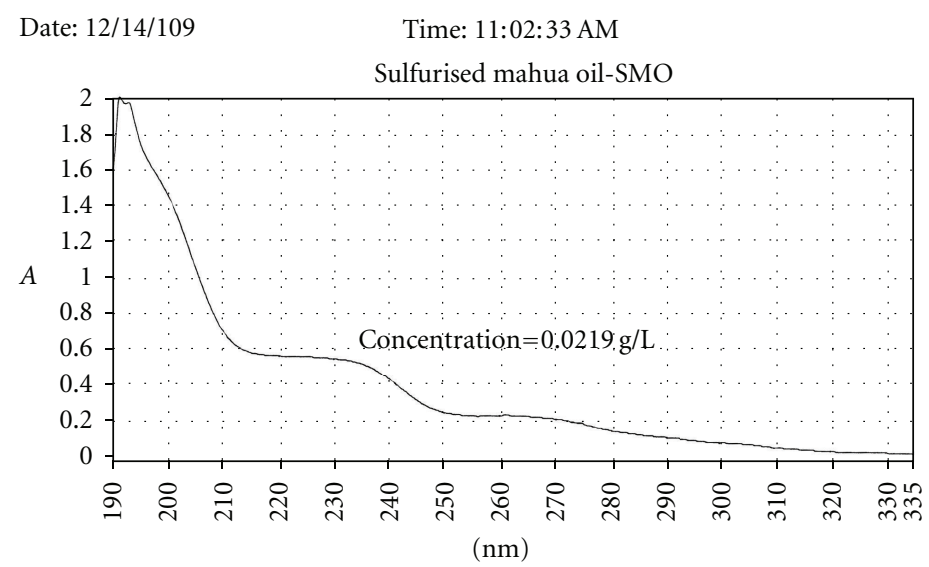

Description:

Spectrum name: C: \UVWINLAB \DATA \EPA-2.SP

Date created: 12/3/09 11:42:27 AM

Instrument model: LAMBDA 19 UV/VIS/NIR

Data interval: $1 \mathrm{~nm}$
Scan speed: $240 \mathrm{~nm} / \mathrm{min}$

Slit width: $1 \mathrm{~nm}$

Smooth bandwidth: $0 \mathrm{~nm}$

FIgURE 4: UV analysis spectrograph of SMO reaction film.

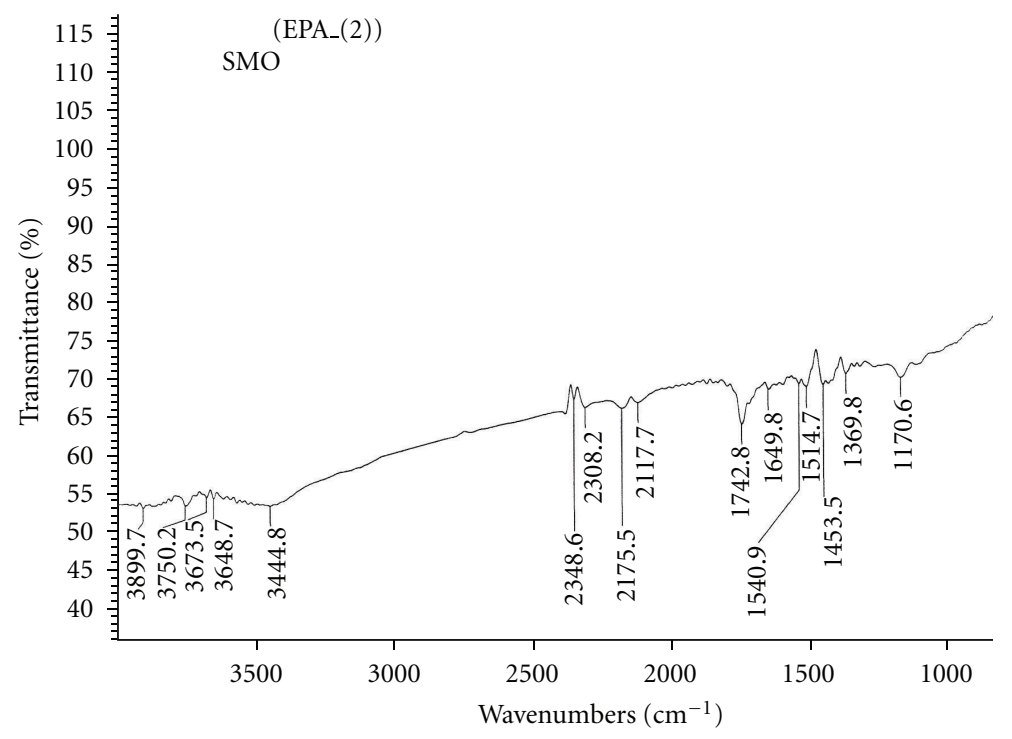

FIGURE 5: FTIR spectrograph of SMO reaction film.

that the composition of film is dependent on the molecular structures of the sulfides. This XRD spectrum of the SMO film is similar to the spectrum of $\mathrm{R}-\mathrm{Fe}-\mathrm{S}-\mathrm{O}$. For the spectrum of SMO film there is only one peak at $44.85 \theta$, but peak intensity is very weak. This information suggests that the reactivity of SMO is higher than that of aromatic sulfide. This SMO decomposed completely and the produced sulfur has been oxidized to iron sulfate on the surface. Oxygen is easily provided by iron on the surface or dissolved oxygen in the oil. It is amorphous in nature.
Film of SMO was analyzed under SEM. The microanalysis in Table 1 conforms the presence of iron. SEM photography was done at 10 places of the SMO film. At places 1, 3 , and 7 it is like sponge structure with gaps. This structure is indicating for the presence of organometallic salt of iron, most probably ferrous sulfate type ( $\mathrm{Fe}-\mathrm{S}-\mathrm{O}-\mathrm{R})$. At places 4 , 5,9 , and 10 it is like stone chips. At places 2, 6, and 8 it is like group of island separated with gaps. So, the film is nonhomogenous and mixture of several types of compounds, for example, crystalline, amorphous, hydrocarbons, and so 


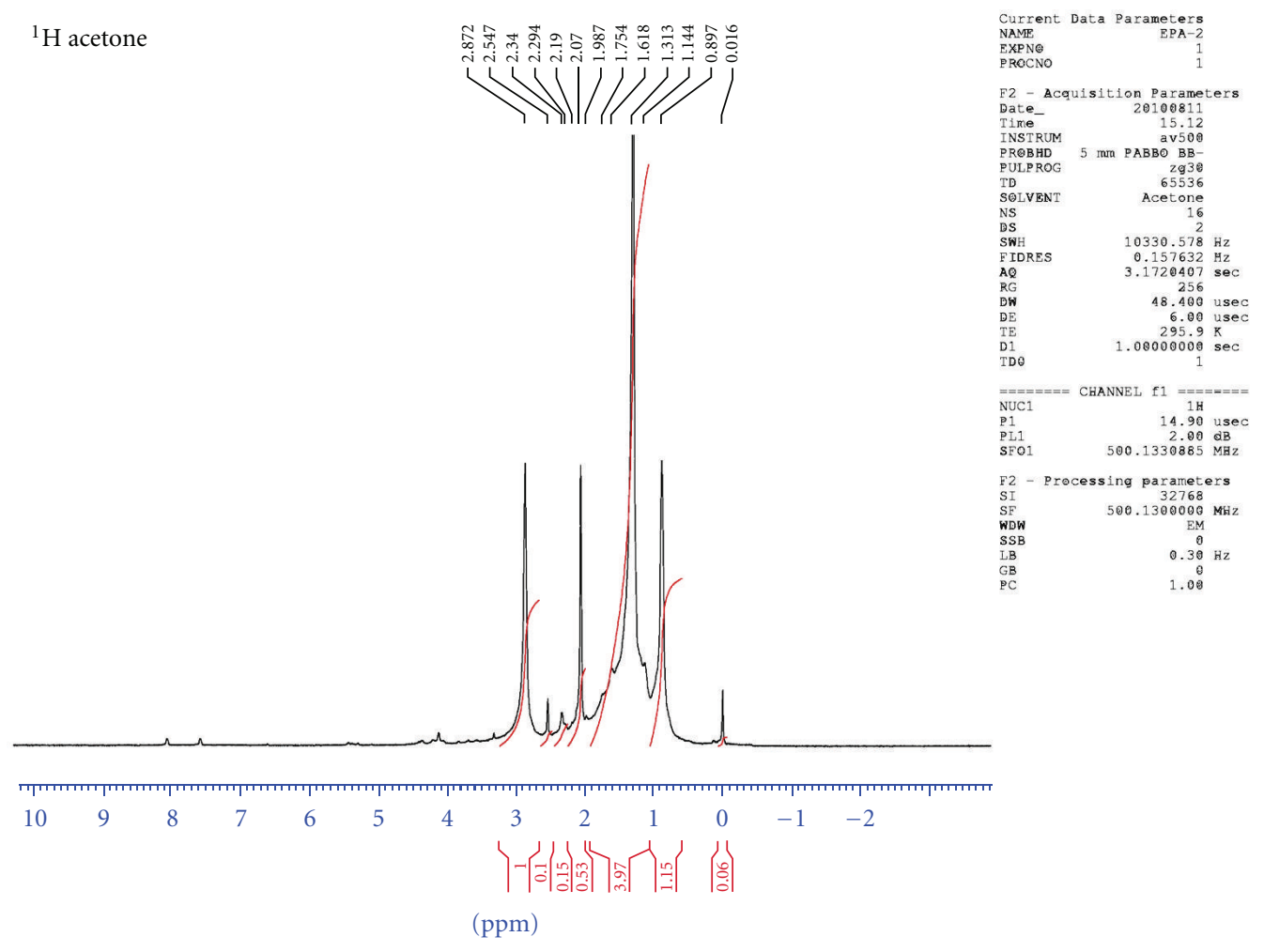

FigURE 6: 1H NMR spectrum of SMO reaction film.

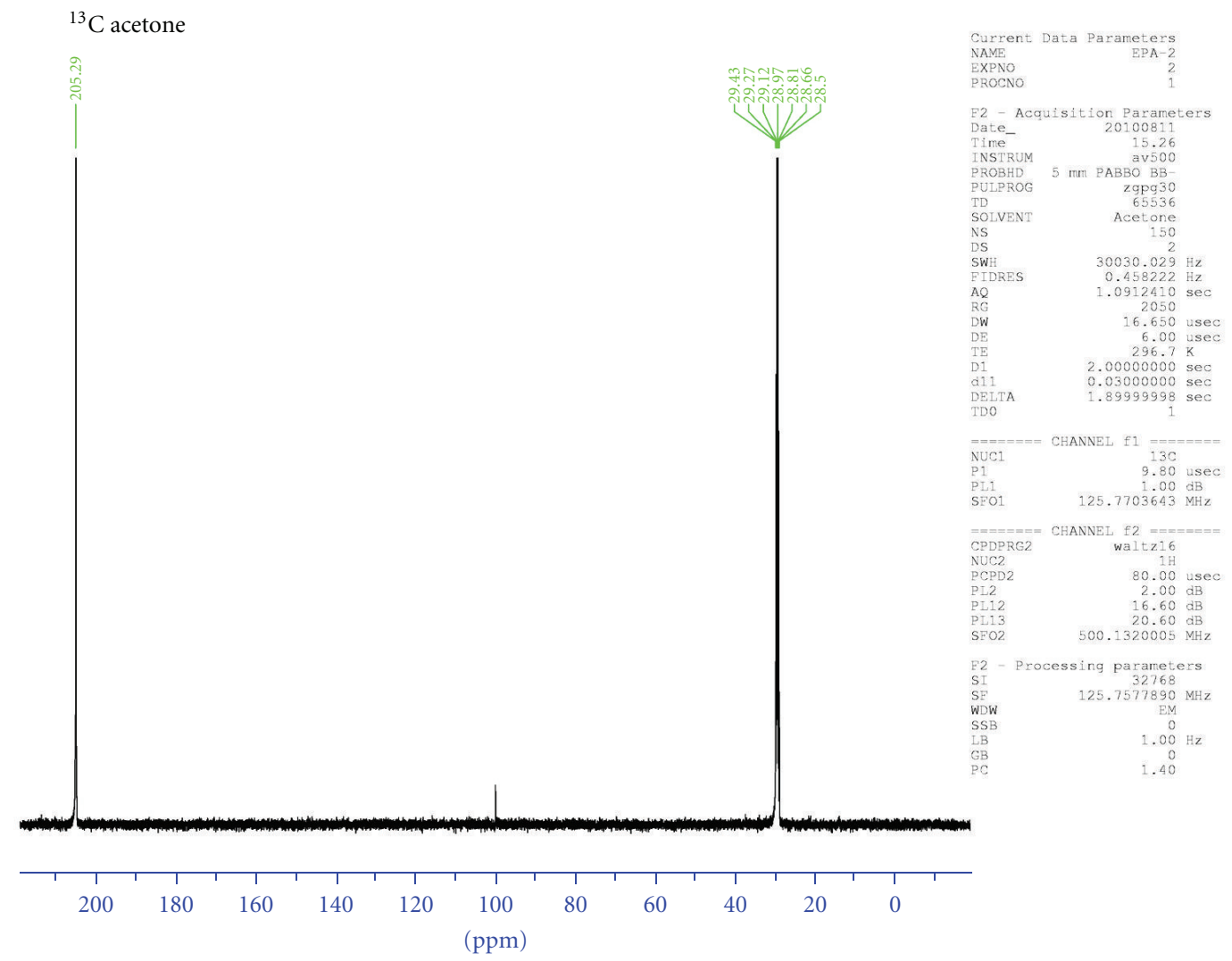

FigURE 7: 13 C NMR spectrum of SMO reaction film. 
EPA2_01.spc 11-May-2010 15:06:15

Chlorite $(\mathrm{Nrm} \%=38.86,20.96,34.83,1.14,3.84,0.28) \quad$ LSecs: 50

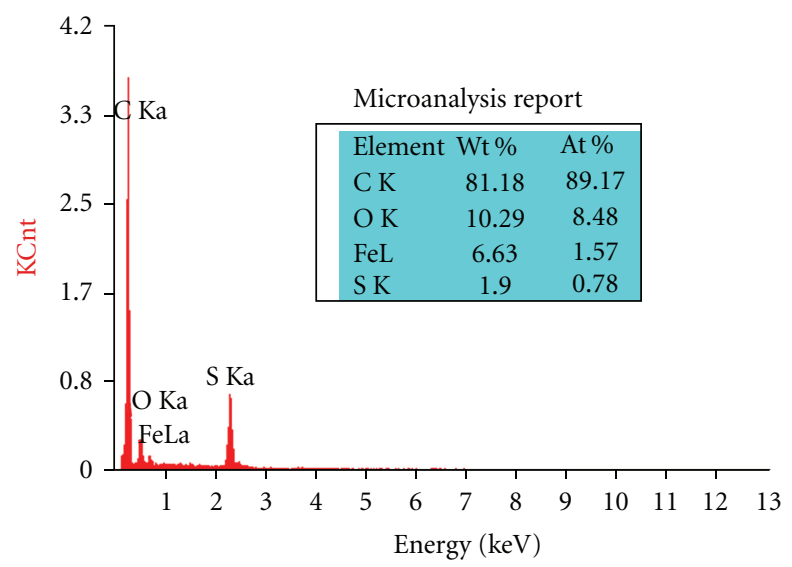

kV: 30 TILT: 0 Take-off: 35 AMPT: 102.4

Detector type: SUTW-Sapphire resolution: 133.44

FIGURE 8: SEM of SMO (EP 2.01).

\EPA2_03.spc 11-May-2010 15:12:13

Chlorite (Nrm\% $=38.86,20.96,34.83,1.14,3.84,0.28) \quad$ LSecs: 50

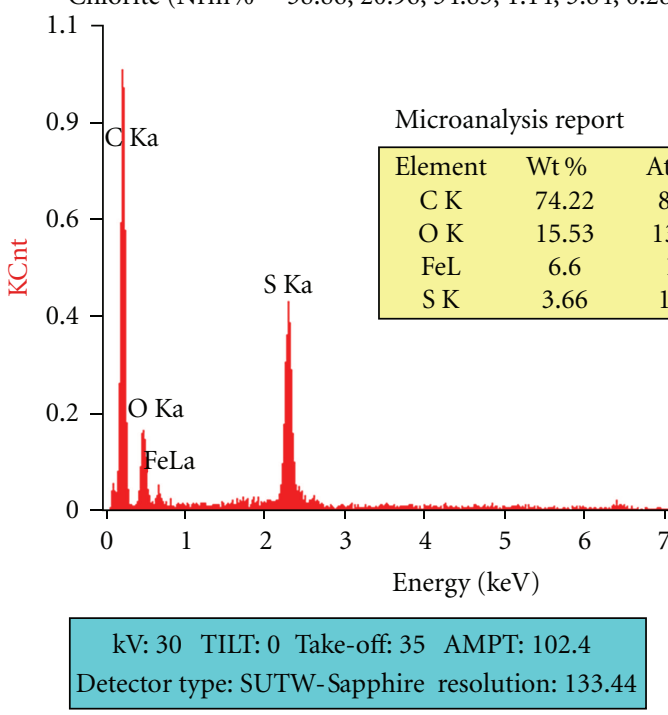

Figure 9: SEM of SMO (EP 2.03).

forth. Film is not continuous. Presence of $\mathrm{FeS}, \mathrm{FeSO}_{4}, \mathrm{Fe}-\mathrm{S}-$ $\mathrm{O}-\mathrm{R}, \mathrm{Ar}-\mathrm{S}$, and so forth is indicated [18].

The $\delta$ values of ${ }^{1} \mathrm{H}$ NMR signals of hydrocarbon portion of SMO film show signals between $\delta 0.16 \mathrm{ppm}$ to $\delta 1.00 \mathrm{ppm}$ is due to the terminal methyl group protons of the hydrocarbon chain of the film. The signal at $\delta 1.14$ to $1.98 \mathrm{ppm}$ due to methylene protons in the alkyl side chain and at $\delta 2.19$ to $2.87 \mathrm{ppm}$ due to methylene protons in alternate side chain. The signals at $\delta 3.97 \mathrm{ppm}$ are due to methylene protons attached to oxygen atom, similar to fatty acid or ester or sulfonate group. The signals at $\delta 2.34 \mathrm{ppm}-\delta 2.54 \mathrm{ppm}$ are due to methine protons. The signal at $\delta 2.07 \mathrm{ppm}$ indicates the presence of unreacted hydrocarbons.
The $\delta$ values of ${ }^{13} \mathrm{C}$ NMR signals of hydrocarbon portion of SMO show signals between $\delta 28.50 \mathrm{ppm}$ to $\delta 28.97 \mathrm{ppm}$ due to the terminal methyl group protons of the alkyl chain. The signal at $\delta 29.12 \mathrm{ppm}$ to $\delta 29.43$ is due to protons of hetero group of alkyl chain. The signal at $\delta 100 \mathrm{ppm}$ is due to protons of hetero group of alkyl $(\mathrm{C}-\mathrm{O}, \mathrm{C}-\mathrm{S})$ and at $\delta$ $205.29 \mathrm{ppm}$ is due to protons near to $\mathrm{C}-\mathrm{O}$ bond of aromatic.

On the sulfurization, sulfur attacks the unsaturation/ double bond of Mohwa oil. Mohwa oil is triglycerides having more than three double bonds and gives polysulfides with sulfur substitution in the allylic position to the double bond. The FTIR analysis of SMO reaction product shows new bands at $1010 \mathrm{~cm}^{-1}, 1020 \mathrm{~cm}^{-1}, 1170 \mathrm{~cm}^{-1}$, 

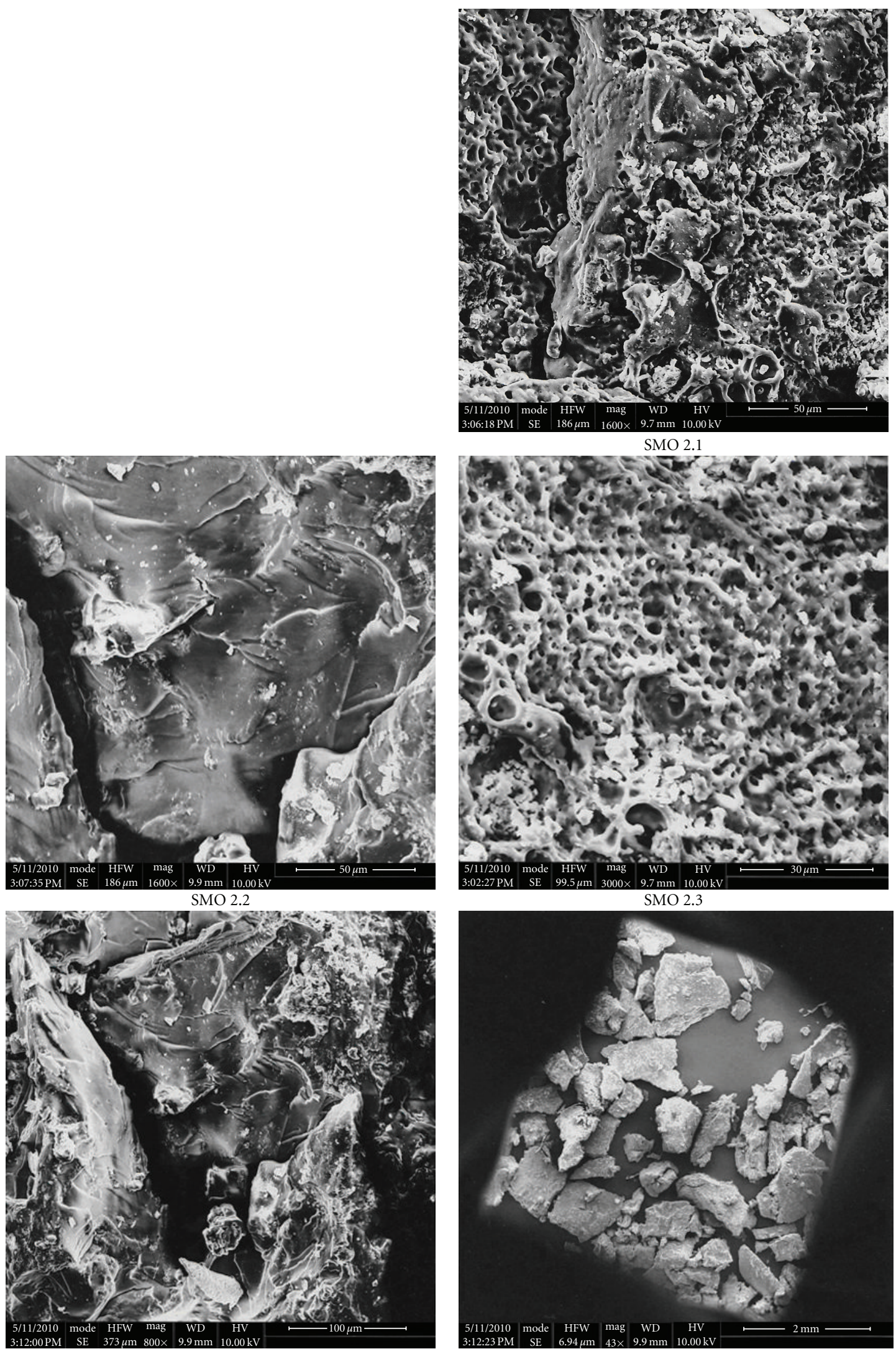

SMO 2.4

SMO 2.5

Figure 10: SEM photo of SMO reaction film. 


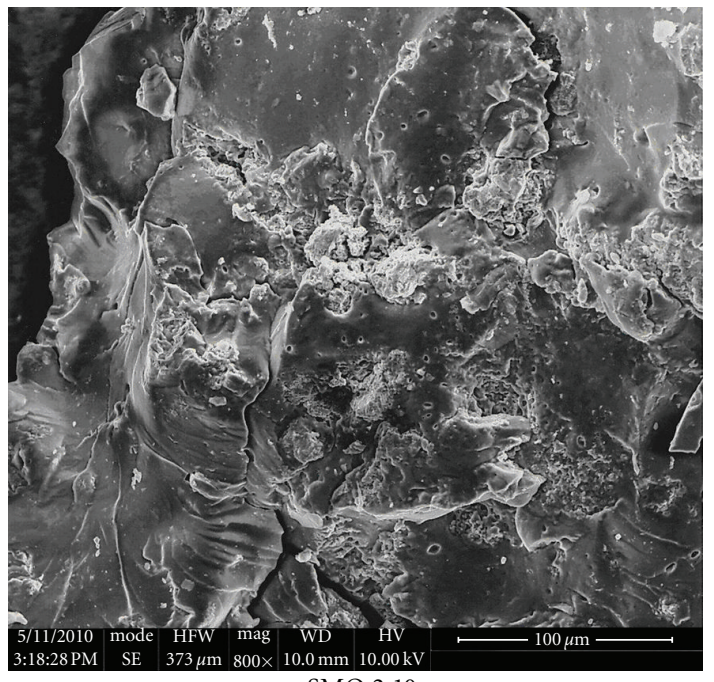

SMO 2.10

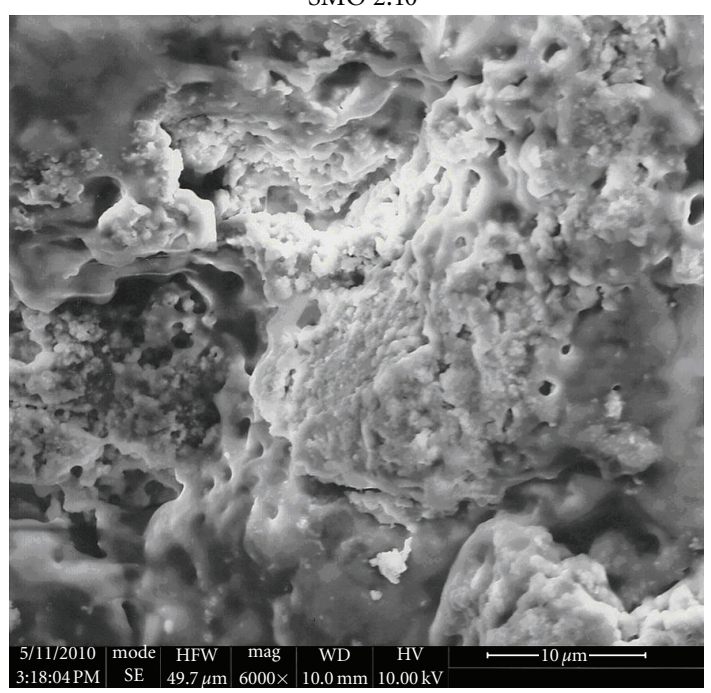

SMO 2.8

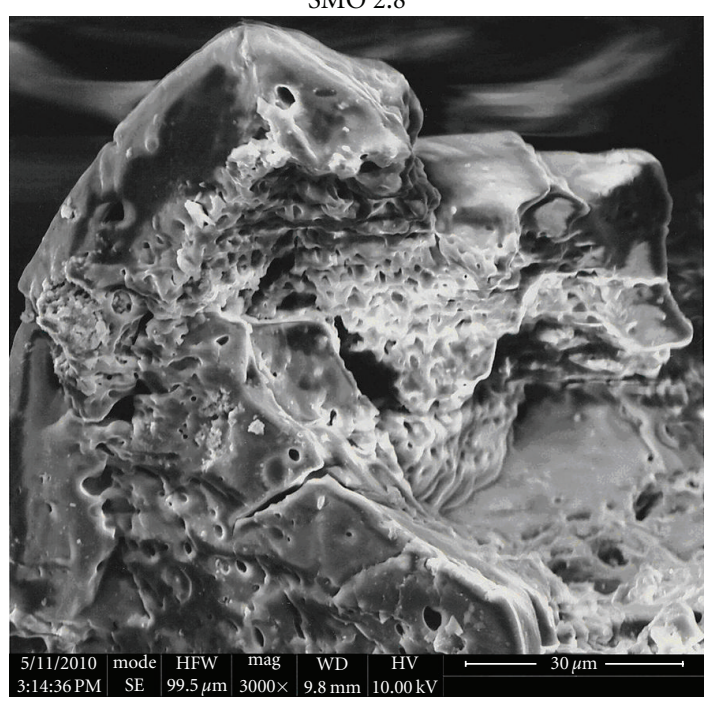

SMO 2.10

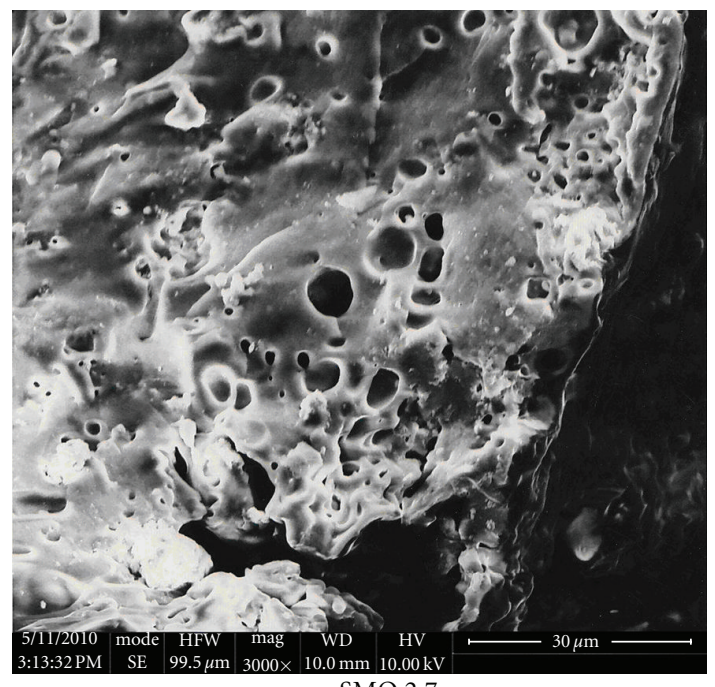

SMO 2.7

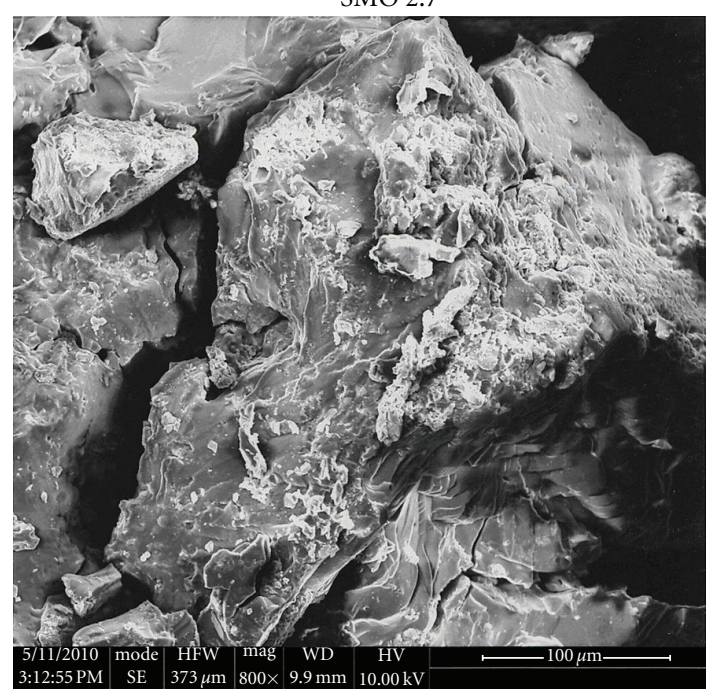

SMO 2.9

FIGURE 11: SEM photo SMO reaction film. 


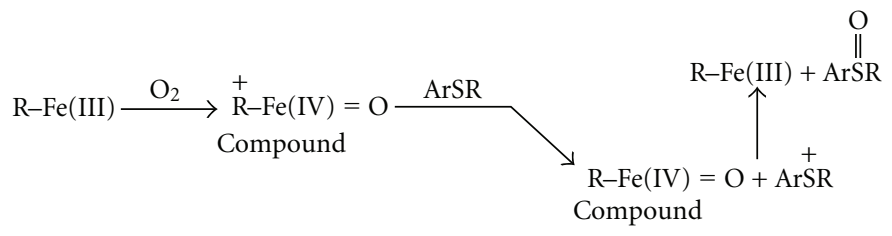

Scheme 2

$1453 \mathrm{~cm}^{-1}, 1514 \mathrm{~cm}^{-1}, 1540 \mathrm{~cm}^{-1}, 1649 \mathrm{~cm}^{-1}, 1742 \mathrm{~cm}^{-1}$, and $3344 \mathrm{~cm}^{-1}$. These are due to ketonic, aldehydic carbonyl, and hydroxyl groups and increase in the unsaturation. It also indicates the elimination of esters and reduction in the length of the alkyl side chain. The reduction in hydrogen carbon ratio indicates the increase in the unsaturation. The changes in elemental composition show an increase in the sulfur content and iron as an additional element. The increase in percentage of oxygen and sulfur shows the presence of carbonyl and hydroxyl group. Band at $3344 \mathrm{~cm}^{-1}$ shows the presence of cyclic ketones and hydroxyl group reduction. This is confirmed by XRD. XRD further indicates the presence of $\mathrm{Fe}-\mathrm{S}-\mathrm{O}$, sulphates, and other inorganics. Film is amorphous in nature and Mohwa oil was fully degraded. UV analysis indicates that major component of cyclic reaction product is monocyclic. The insignificant change in hydrogen carbon ratio indicates that sulfoxides are produced rather than oxidation of hydrocarbon. NMR indicates that organic sulphide or sulfoxides are present and $\mathrm{S}-\mathrm{O}, \mathrm{C}-\mathrm{O}$ or $\mathrm{C}-$ $\mathrm{S}$ linkage is present. The SEM indicates for the presence of organic-inorganic mixture with unreacted hydrocarbon solvent. The film is nonhomogenous and mixture of all types of compounds, for example, crystalline, amorphous, hydrocarbons, and so forth. Film is not continuous. So, it may be possible that $\mathrm{FeS}, \mathrm{FeSO}_{4}, \mathrm{Fe}-\mathrm{S}-\mathrm{O}-\mathrm{R}, \mathrm{Ar}-\mathrm{S}$, and so forth are present. The probable sequence of reaction, indicated from the analysis, involves the reaction of iron on the S-S bond, which leads to formation of iron sulfides and elimination of ester group along with oxidation of the alkyl side chain to form ketone, aldehyde, and carboxylic group (giving FTIR band at 1540 and $1170 \mathrm{~cm}^{-1}$ ) by oxidation. The prominent reaction is oxidation to convert sulfide group into aldehyde to carboxylate and then formation of polymeric iron carboxylate film. The film formed from SMO has an unsaturated cyclic ring system which is nonaromatic in nature, therefore, the strength of the film formed by the SMO is greater.

In the case of these films, sulfur is present in several oxidation states as indicated by XRD peaks. The peaks could be attributed to oxidized form of sulfur as the sulfoxide. The XRD spectrum for SMO with the appearance of only one or no peak corresponds to iron sulfate. And the relative intensity of SMO is stronger. This means the bulk of film generated from the additives is composed of $\mathrm{Fe}-\mathrm{S}-$, and the bulk layer of SMO is thinner. The produced RS - or RSS - radical of alkyl polysulfide reacted with the metal or iron oxide to form $\mathrm{FeSO}_{4}$. The higher sulfur content in the sulfides leads to the higher content of radical, accelerating the rate of reaction. As to the inner surface of the film, the content of iron oxide and oxygen decreases, which results in the variation of the film composition, that is, the bulk consists of $\mathrm{FeSO}_{4}$, subsurface consists of a mixture of $\mathrm{FeSO}_{4}$, sulfoxide, and $\mathrm{FeS}_{2}$, and the out surface consists of adsorbed alkyl disulfide. For the SMO, due to the polar group in the molecular structures, it is adsorbed strongly onto the metal surface and a thick protective film formed, which hinders the reaction of mercaptyl radical with the metal surface. So a film consisting of $\mathrm{FeS}_{2}$ from the bulk to the subsurface is formed. Therefore, $\mathrm{FeS}_{2}$ is one of the most probable forms of sulfides in the film. $\mathrm{Fe}_{3} \mathrm{~S}_{4}$, which can be the same crystal system as $\mathrm{Fe}_{3} \mathrm{O}_{4}$, is another candidate although it is a less common compound. This is confirmed by XRD. XRD further indicates the presence of $\mathrm{Fe}-\mathrm{S}-\mathrm{O}$, sulphates, and other inorganics. Film is amorphous in nature. UV analysis indicates that major component of cyclic reaction product is monocyclic. The insignificant change in hydrogen carbon ratio indicates that sulfoxides are produced instead of oxidation of hydrocarbon. NMR indicates that organic sulphide or sulfoxides are present and $\mathrm{S}-\mathrm{O}$, $\mathrm{C}-\mathrm{O}$ or $\mathrm{C}-\mathrm{S}$ linkage is present. SEM indicating for the presence of organic-inorganic mixture with unreacted hydrocarbon solvent. The film is nonhomogenous and mixture of all types of compounds, for example, crystalline, amorphous, hydrocarbons, and so forth. Film is not continuous. Presence of $\mathrm{FeS}, \mathrm{FeSO}_{4}, \mathrm{Fe}-\mathrm{S}-\mathrm{O}-\mathrm{R}, \mathrm{Ar}-\mathrm{S}$, and so forth are indicated.

The oxygen atom transfer (electron transfer) mechanism revealed that the iron-oxo complex radical cation, $\mathrm{Fe}(\mathrm{IV})=\mathrm{O}$, is active oxidant. When the oxidation of a phenyl/alkyl sulfides occured the corresponding sulfoxides were produced. Use of $\mathrm{H}_{2}{ }^{18} \mathrm{O}$ or $\mathrm{H}_{2}{ }^{18} \mathrm{O} 2$ clearly indicates that the oxygen atom in the sulfoxides comes exclusively from the oxidant. Moreover, no fragmentation products were observed in the oxidation of an SMO whose radical cation is expected to undergo cleavage of the $\beta \mathrm{C}-\mathrm{H}$ and $\mathrm{C}-\mathrm{S}$ bonds. These results would seem to suggest a direct oxygen atom transfer from the iron-oxo complex to the sulfide. The oxygen incorporation indicates that the intermediate radical cation, once formed, has to react with $\mathrm{RFe}(\mathrm{IV})=\mathrm{O}$ (the reduced form of the ironoxo complex which is formed) in a fast oxygen rebound (Scheme 2) [19].

Several authors erroneously regard SMO as possessing inferior surface protector properties despite the fact that experimental surface properties data obtained by several workers and at the authors' laboratory quite clearly show that the surface characteristics of SMO are not inferior and comparable with those of lower SMO at equivalent sulfur concentrations up to $0.5 \%$. At equivalent sulfur concentrations above $0.75 \%$ the surface properties are definitely superior. This contention has arisen because SMO gives the least amount of iron sulfide. On the other hand, the above results 
are consistent with the observation that the decomposition of $\mathrm{SMO}(35 \mathrm{wt} \%)$ at $150^{\circ} \mathrm{C}$ for 8 hours in the presence of hydrocarbon solvent is about $10 \mathrm{wt} \%$ higher than that of dibenzyl disulfide (DBDS) at $1 \%$ equivalent sulfur concentration [2022].

\section{Conclusion}

On the basis of the studies, the following conclusions can be drawn.

(1) The sequence of reactions taking place on the rubbing surface involved the reaction of iron with the S-S bonds to form from iron sulfide, with simultaneous oxidation and elimination of alkyl chains, finally leading to the formation of polymeric film with repeating units of unsaturated cyclic rings joined together by iron sulfide functional group. The nature of the ring system produced from the sulfurized Mohwa oil is nonaromatic.

(2) The surface protective capacities of the various sulfides depend upon the combined strength of the organic film and the iron sulfide formed. The structural constituents of the organic film profoundly influence its strength. The film which is aromatic in nature has better protective capacities than the nonaromatic one.

(3) The films formed are organo-inorganic in nature. A polymeric organic film is superimposed on inorganic iron sulfide.

(4) A variety of strongly absorbed organic species are formed as films on metal surfaces under various chemical environments owing to interaction between the organic/inorganic sulfides, hydrocarbon media and metal.

(5) The quantity and quality of these organic films have profound protective properties. Films containing aryl derivatives of iron possess better protective capacities than the corresponding alkyl derivatives.

(6) The quantity and composition of iron sulfide alone do not seem to have a crucial property than aryl disulfides.

(7) The strength of the C-S and S-S bonds and the reactivity of the subsequent radicals produced with iron have a pronounced protection on the metal surface.

(8) The films formed from the sulfides are mainly composed of Fe-S-O, and alkyl disulfide also exists in the subsurface and bulk of film generated from alkyl sulfides. The film generated from the SMO is composed of alkyl disulfides in the out surface, a mixture of $\mathrm{FeSO}_{4}$, sulfoxide and $\mathrm{FeS}_{2}$ in the subsurface, and $\mathrm{FeSO}_{4}$ in the bulk.

\section{References}

[1] C. Kajdas, "Importance of anionic reactive intermediates for lubricant component reactions with friction surfaces," Lubrication Science, vol. 6, no. 3, pp. 203-228, 1994.

[2] E. H. Loeser, R. C. Wiquist, and S. B. Twiss, "Cam and tappet lubrication III-radioactive study of phosphorus in the EP film," Transactions of American Society of Lubrication Engineers, vol. 1, no. 2, pp. 329-335, 1958.

[3] W. Davey and E. D. Edwards, "The extreme-pressure lubricating properties of some sulphides and disulphides, in mineral oil, as assessed by the Four-Ball Machine," Wear, vol. 1, no. 4, pp. 291-304, 1958.

[4] D. Godfrey, "Chemical changes in steel surfaces during extreme pressure lubrication," Transactions of American Society of Lubrication Engineers, vol. 5, no. 1, pp. 57-66, 1962.

[5] T. Sakurai and K. Sato, "Study of corrosivity and correlation between chemical reactivity and load-carrying capacity of oils containing extreme pressure agent," Transactions of American Society of Lubrication Engineers, vol. 9, pp. 77-87, 1966.

[6] K. G. Allum and E. S. Forbes, "The load-carrying mechanism of organic sulfur compounds-application of electron probe microanalysis," Transactions of American Society of Lubrication Engineers, vol. 22, no. 2, pp. 162-175, 1968.

[7] R. C. Coy and T. F. J. Quinn, in Proceedings of the IMechE Conference on Lubrication, September 1973.

[8] A. Dorinson and V. E. Broman, "Extreme pressure lubrication and wear. The chemical reactivity and the extreme pressure action of two aliphatic disulfides," Transactions of American Society of Lubrication Engineers, vol. 5, no. 1, pp. 75-90, 1962.

[9] F. T. Barcroft, "A technique for investigating reactions between E.P. additives and metal surfaces at high temperatures," Wear, vol. 3, no. 6, pp. 440-453, 1960.

[10] T. Sakurai, K. Sato, and K. Ishida, "Reaction between sulfer compounds and metal surfaces at high temperatures," Bulletin of The Japan Petroleum Institute, vol. 6, pp. 40-46, 1964.

[11] K. G. Allum and J. F. Ford, "The influence of chemical structure on the load currying properties of certain organo sulphur compounds," Journal of the Institute of Petroleum, vol. 51, pp. 145-161, 1965.

[12] E. S. Forbes, "The load-carrying action of organo-sulphur compounds-a review," Wear, vol. 15, no. 2, pp. 87-96, 1970.

[13] F. G. Rounds, "Effects of additives on the friction of steel on steel I. Surface topography and film composition studies," Transactions of American Society of Lubrication Engineers, vol. 7, no. 1, pp. 11-23, 1964.

[14] S. B. Crecetus and R. E. Kagrise, "Drying oil oxidation mechanism, film formation, and degradation-enthalpy, entropy, and isobaric heat capacity at $100^{\circ}$ to $1000^{\circ} \mathrm{C}$. and 50 to 1400 bars," Journal of Industrial and Engineering Chemistry, vol. 47, no. 8, pp. 1643-1649, 1955.

[15] O. N. Anand, J. Mehta, and T. S. R. Rao Prasada, "Lubricant components from vegetable oils of Indian origin," Journal of Synthetic Lubrication, vol. 5, no. 2, pp. 99-106, 1998.

[16] J. H. Skellon and M. N. Thruston, "The oxidation of monoethenoid fatty acids and esters. Union of gaseous oxygen with elaidic acid, and methyl and n-propyl elaidate," Journal of the Chemical Society, pp. 1626-1630, 1949.

[17] O. N. Anand, V. P. Malik, K. D. Neemla, and R. P. S. Bisht, in Proceedings of the $3 r d$ Symposium on Lubricants -AdditivesWaxes and Petroleum Speciality Products (LAWPSP '83), Bombay, India, January 1983.

[18] K. Ramaswamy, M. A. Malik, M. Helliwell, F. Tun, and P. O'Brien, "Iron thiobiurets: single-source precursors for iron 
sulfide thin films," Inorganic Chemistry, vol. 49, no. 18, pp. 8495-8503, 2010.

[19] S. Plaza, ASLE Transactions, vol. 28, pp. 21-30, 1987.

[20] W. A. Pryor, Mechanism of Sulfur Reaction, McGraw-Hill, New York, NY, USA, 1962.

[21] M. Venkataramanan, G. Skanth, K. Bandyopadhyay, K. Vijayamohanan, and T. Pradeep, "Self-assembled monolayers of two aromatic disulfides and a diselenide on polycrystalline silver films: an investigation by sers and XPS," Journal of Colloid and Interface Science, vol. 212, no. 2, pp. 553-561, 1999.

[22] A. Kassim, T. W. Tee, D. K. Abdullah et al., "Synthesis and characterization of crosslinked polyacrylamide (PAAM)-carrageenan hydrogels superbasorbent prepared by gamma radiation," Indonesian Journal of Chemistry, vol. 10, no. 1, pp. 8-11, 2010. 

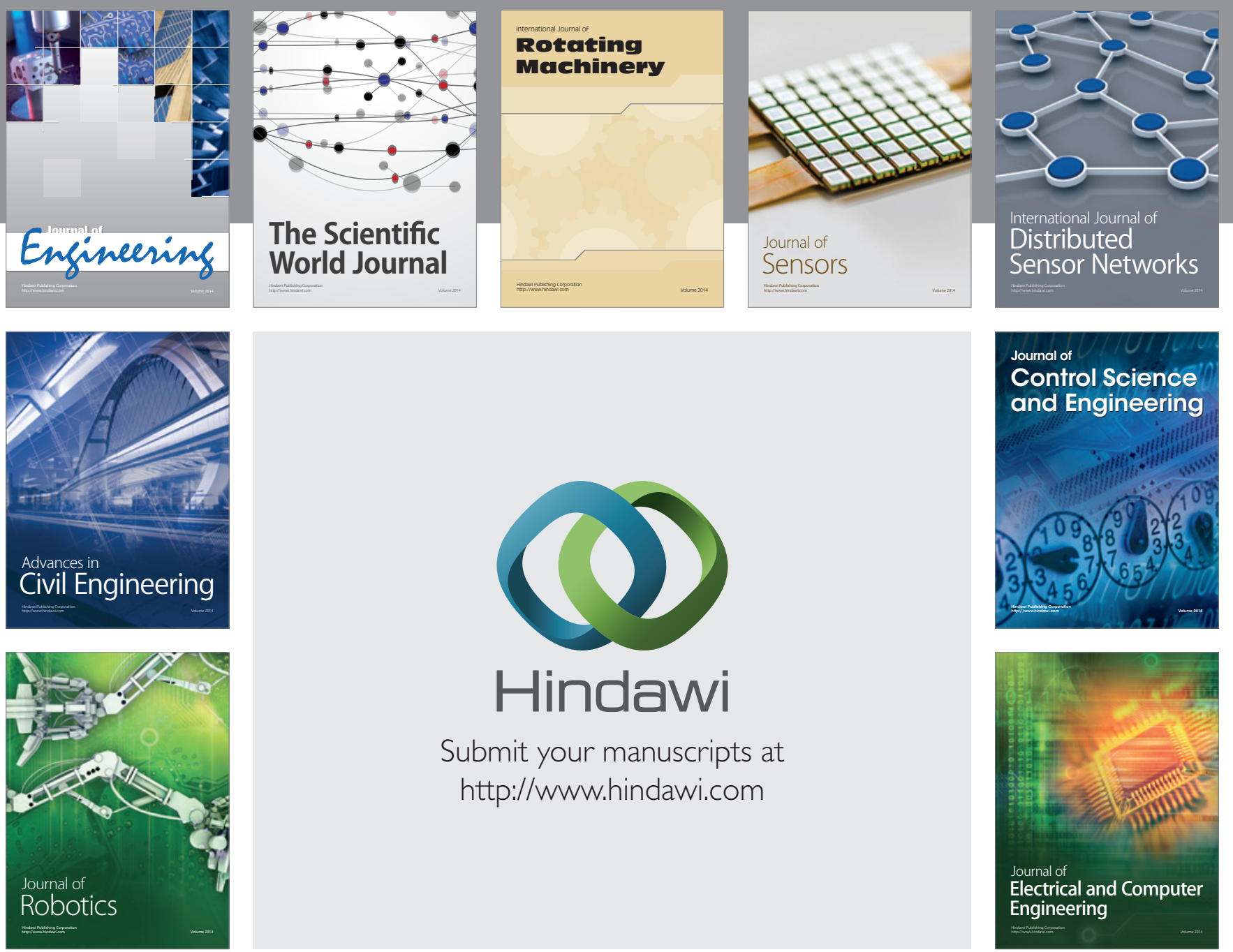

Submit your manuscripts at

http://www.hindawi.com
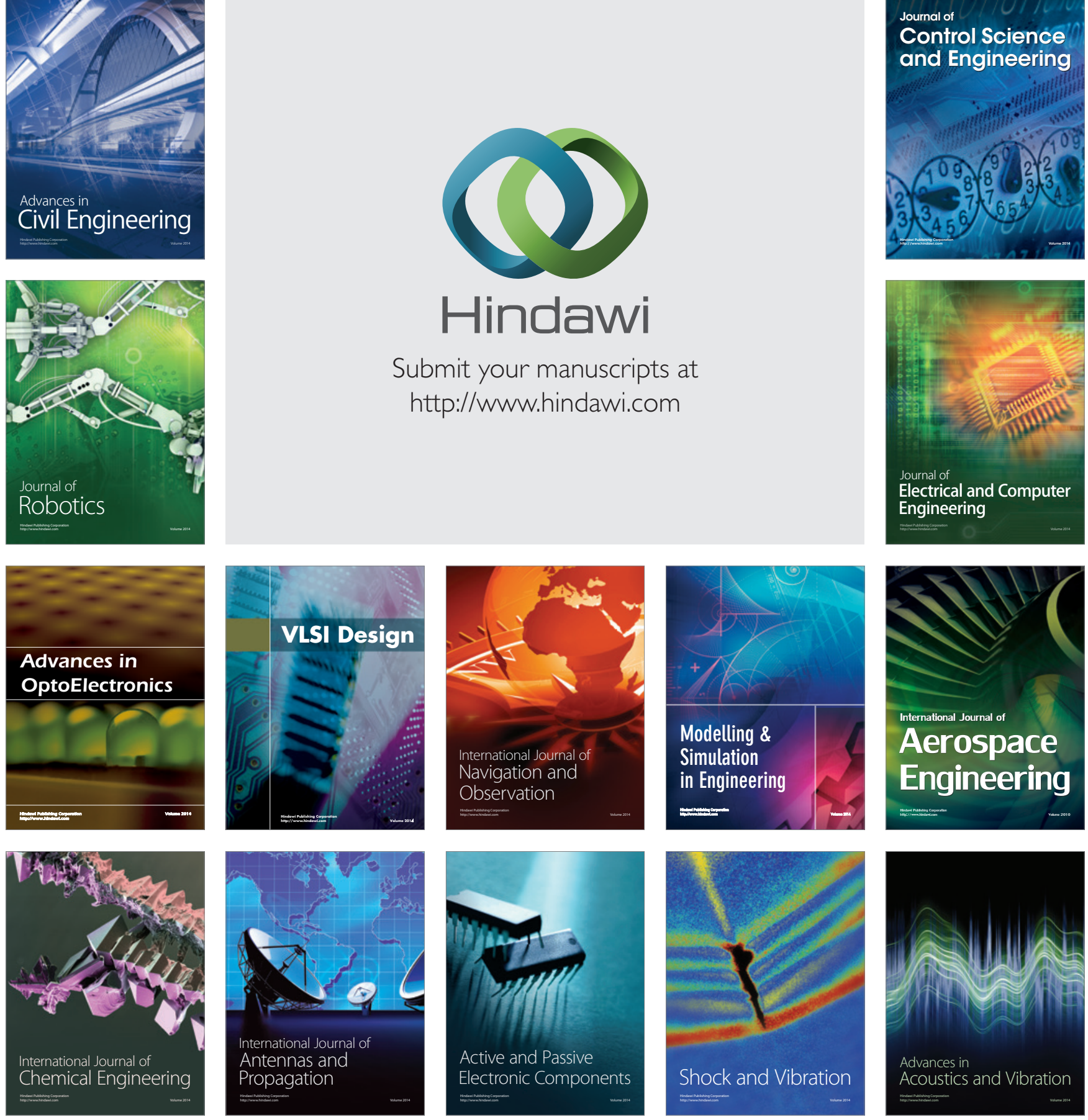